\title{
Adaptive model predictive control for linear time varying systems
}

\author{
Marko Tanaskovic $^{\mathrm{a}}$, Lorenzo Fagiano ${ }^{\mathrm{b}}$, Vojislav Gligorovski ${ }^{\mathrm{c}}$ \\ ${ }^{\text {a }}$ Singidunum University, Belgrade, Serbia. \\ ${ }^{\mathrm{b}}$ Dipartimento di Elettronica, Informazione e Bioingegneria, Politecnico di Milano, Milano, Italy \\ ${ }^{\mathrm{c}}$ Laboratory of the physics of biological systems, Swiss Federal Institute of Technology Lausanne (EPFL)
}

\begin{abstract}
A robust, adaptive Model Predictive Control (MPC) approach for asymptotically stable, constrained linear time-varying (LTV) systems with multiple inputs and outputs is proposed. The approach consists of two-steps, carried out on-line with a receding horizon strategy. In the first one, a real-time Set Membership identification algorithm exploits the measured input-output data and the available prior knowledge to build and refine a set of admissible models of the plant (Feasible Parameter Set, FPS). This set is guaranteed to contain also the true system dynamics under the considered working assumptions. In the second step, a robust finite-horizon optimal control problem is formulated and solved. The variation of system dynamics is taken into account by inflating the FPS over the prediction horizon, according to worst-case bounds, assumed a-priori, on the parameters' rate of change. The resulting optimal control sequence guarantees that the outputs of all possible plants inside the FPS satisfy the operational constraints, also considering all possible future parameter changes. The main theoretical properties of the proposed approach are demonstrated and the method is showcased in numerical simulations, highlighting the fundamental improvement over previous approaches not designed for LTV systems.
\end{abstract}

Key words: Adaptive control, Learning control, LTV systems, Set membership identification, Model predictive control, Constrained control

\section{Introduction and motivation}

MPC is a successful technique in industrial applications, thanks to the capability to explicitly account for constraints on inputs and outputs and to incorporate information on future disturbance and reference signals [16]. A research direction that is currently receiving growing interest is that of dual, adaptive and learning-based MPC, i.e. approaches where the model derivation/identification step is considered together with the control computation, and possibly carried out on-line. There are several contributions that differ in terms of system dynamics (linear or nonlinear), uncertainty characterization (stochastic or unknown-but-bounded), and model identification scheme (off-line or on-line/adaptive), see e.g. [1], [2], [3], [4], [8], [11], [12], [13], [14], [18], [22], [23], [15],[10]. These works are motivated by the difficulty to derive models based on physical principles for complex processes, the increasing real-time availability of measured data, and the want to derive MPC approaches that can automatically adapt to uncertain and time-varying dynamics.

Set Membership (SM) techniques are being adopted by several researchers for the model identification phase, since they provide, in addition to a nominal model of the plant, a

Email addresses: mtanaskovicesingidunum.ac.rs (Marko Tanaskovic), lorenzo.fagiano@polimi.it (Lorenzo Fagiano), vojislav.gligorovskidepfl.ch (Vojislav Gligorovski). quantification of the associated uncertainty, which can be exploited for MPC design. Examples of contributions exploiting SM techniques are [4],[12], [13] and [18]. In [18], we proposed the use of SM identification to derive an adaptive MPC approach for uncertain linear time-invariant systems, subject to both process disturbance and measurement noise. This approach guarantees robust constraint satisfaction on the outputs also during model adaptation, which is a major challenge in adaptive control under constraints. However, it does not provide the same guarantees in presence of timevarying dynamics: indeed the method can easily fail in this case, as we show here through a motivating example. The main contribution of this paper is to remove this deficiency, through a modified adaptive technique that can cope with LTV systems. As in [18], a two-step procedure is employed. In the first step, a set of models consistent with measured data and prior assumptions is built and refined (Feasible Parameter Set, FPS). This set is guaranteed to contain also the true system dynamics, under the considered working assumptions. Differently from [18], we now take into account the time-varying nature of the plant, both in the future predictions and in how the past data is exploited to build the FPS. In the second step, a robust finite-horizon optimal control problem is formulated and solved, where we also predict all possible future changes of the model set. The procedure is implemented on-line with a receding horizon strategy. We prove that the resulting feedback control law guarantees recursive feasibility and robust constraint satisfaction, and il- 
lustrate its performance in the motivating example, showing that the new approach removes efficiently a fundamental limitation of the previous one. To the best of our knowledge, to date there are no other adaptive techniques in the literature providing similar theoretical guarantees in presence of output constraints, system uncertainty, process disturbance, and measurement noise.

\section{Motivating example}

Consider an LTV, single-input, single-output mass-springdamper system with natural frequency equal to 1 , gain equal to 4 , and eigenvalues in the left half plane with damping ratio $\xi$. The value of $\xi$ can change over time inside the interval $[0.3,1]$. The amplitude of the input $u$ is constrained in the compact set $[-1.5,1.5]$ and its rate of change $\Delta u$ in $[-0.5,0.5]$. By discretizing the dynamics with the trapezoid method and sampling time $T_{s}=0.7 \mathrm{~s}$ and truncating the system's infinite impulse response (IIR) at $m=24$ samples, one obtains the Finite Impulse Response (FIR) coefficients reported as an example in Fig. 1 for the values $\xi=0.3$ and $\xi=1$.

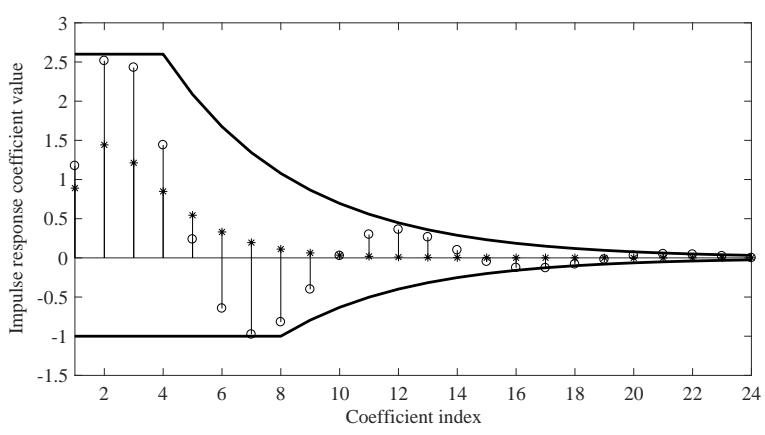

Fig. 1. Impulse response of the LTV plant with $\xi=1$ (stars) and $\xi=0.3$ (circles), and considered set $\Omega$ (thick black lines).

The truncated part of the impulse response is embedded into an additive disturbance signal $d$, acting on the output $y$. Such a disturbance is bounded, because of the bounded input and the fact that, for any fixed value of $\xi$, the corresponding LTI system is asymptotically stable, i.e. its impulse response decays exponentially to zero. In the described settings, the exact bound on this additive contribution is 0.1 . We further assume that an exogenous additive disturbance affects the output, with maximum amplitude 0.05. Thus, the total bound on the additive output disturbance $d$ is 0.15 . The system dynamics are not known exactly a priori, but noise-corrupted measurements of the output are available, with bounded noise $v:|v| \leq 0.1$. As prior information, we assume that the FIR coefficients belong to a polytopic set $\Omega$, bounded by the thick black lines in Fig. 1, and that the bounds on the additive disturbance and measurement noise are equal to 0.2 and 0.15 , respectively. Note that this prior information is not tight, i.e. the signal bounds and the polytopic set are not known exactly. The control problem is to track a given reference output, while satisfying the output constraints $y \in[-6,6]$. The change of $\xi$ over time in our simulation test is shown in Fig. 2.

In [18], we proposed an adaptive MPC technique based on
SM identification for uncertain LTI plants, able to robustly enforce output constraints also during adaptation. One of the core steps in this approach is the real-time refinement of the FPS, i.e. the set of all models compatible with the collected measurements and prior assumptions. When applied to the LTV case, the approach of [18] looses its theoretical guarantees and in practice it can easily fail, as shown in Fig. 3 , where at time step $k=42$ the FPS becomes empty due to the inconsistency between the collected data, produced by the change of system dynamics. This problem can occur with rather small changes of the plant (3\% reduction of $\xi$ in this example), and it does not depend on the parameters' rate of change: also with very slow variations there is eventually an instant when an inconsistency among the past data can arise. Finally, note again that the assumed initial set $\Omega$ containing the unknown system parameters, i.e. the starting FPS, covers all possible FIR coefficients of the true system (see Fig. 1). Thus, the problem is neither due to the parameters being outside the assumed initial bounds. Rather, it is caused by a fundamental limitation of the adaptive MPC approach of [18] when applied to LTV plants. On the one hand, this effect could be used for condition monitoring and fault detection schemes, since emptiness of the FPS indicates a change in the plant dynamics. On the other hand, we present here an approach that removes such a limitation, restoring the recursive feasibility and constraint satisfaction guarantees achieved in [18].

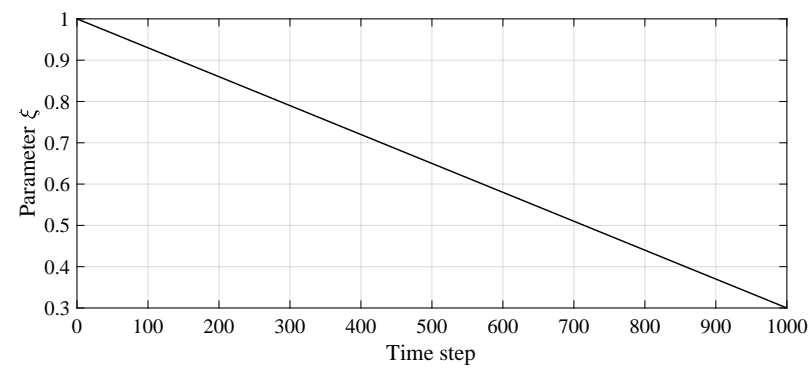

Fig. 2. Change of damping parameter $\xi$ over time. At $k=42$ the value of $\xi$ is $0.97,3 \%$ smaller than the starting value.

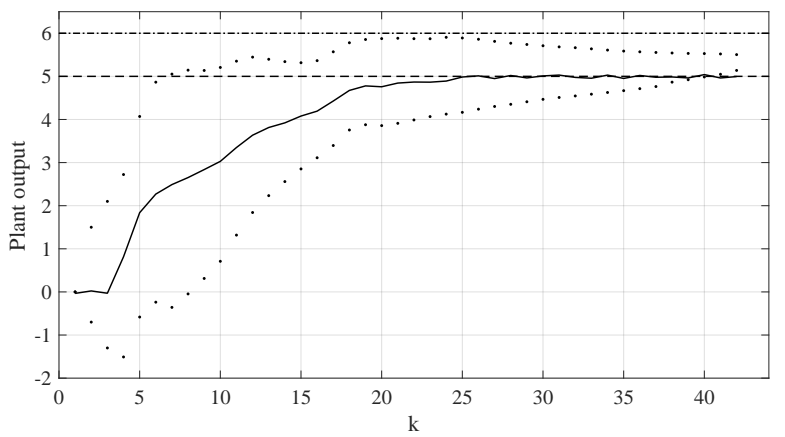

Fig. 3. Plant output (thick black line), reference (dashed), uncertainty bounds (dotted) and constraints (dash-dotted) when the adaptive MPC algorithm from [18] is used. At $t=42$, the output exits the uncertainty range defined by the FPS, which becomes empty at the next step causing the control algorithm to fail. 


\section{Problem Statement}

We consider a discrete-time LTV system with $n_{u}$ inputs and $n_{y}$ outputs. The system at each time step $t \in \mathbb{Z}$ is known to be asymptotically stable, but the exact dynamics and the way they change over time are not known precisely. We denote the vector of control inputs by $u(t)=\left[u_{1}(t), \ldots, u_{n_{u}}(t)\right]^{T}$, where $u_{i}(t) \in \mathbb{R}, i=1, \ldots, n_{u}$ are the individual plant inputs and ${ }^{T}$ stands for the matrix transpose operator. Similarly, we denote the vector of plant outputs by $y(t)=$ $\left[y_{1}(t), \ldots, y_{n_{y}}(t)\right]^{T}$. We consider a family of models for this system, whose equations read:

$$
y_{j}(t)=H_{j}^{T}(t) \varphi(t)+d_{j}(t), j=1, \ldots, n_{y} .
$$

In (1), the vector $d(t)=\left[d_{1}(t), \ldots, d_{n_{y}}(t)\right]^{T}$ accounts for exogenous additive disturbances and the effects of neglected dynamics on the outputs (e.g. due to truncation of the IIR of the system, see Remark 3.1). $\varphi(t) \in \mathbb{R}^{m}$ is a regressor vector with $m$ elements, that evolves over time according to the following linear model:

$$
\varphi(t+1)=F \varphi(t)+G u(t)
$$

where matrices $F \in \mathbb{R}^{m \times m}$ and $G \in \mathbb{R}^{m \times n_{u}}$ depend on the chosen model parametrization. For example, when $n_{u}=1$ and a Finite Impulse Response (FIR) plant model is used, $F$ and $G$ have the following structure:

$$
F=\left[\begin{array}{ccccc}
0 & 0 & \ldots & 0 & 0 \\
1 & 0 & \ldots & 0 & 0 \\
\vdots & \vdots & \ddots & \vdots & \vdots \\
0 & 0 & \ldots & 1 & 0
\end{array}\right], G=\left[\begin{array}{c}
1 \\
0 \\
\vdots \\
0
\end{array}\right]
$$

For the case $n_{u}>1, F$ and $G$ can be obtained by block diagonalizing the matrices in (3). Moreover, suitable $F$ and $G$ matrices can be derived for Laguerre [20], Kautz [21] or generalized basis functions [6] parameterizations.

Each of the vectors $H_{j}(t) \in \mathbb{R}^{m}$ in (1) contains the model parameters that describe the influence of $\varphi$ to the plant output $j$ at time step $t$. Defining the matrix $H(t) \in \mathbb{R}^{n_{y} \times m}$ as $H(t) \doteq\left[H_{1}(t), \ldots, H_{n_{y}}(t)\right]^{T}$, we have:

$$
y(t)=H(t) \varphi(t)+d(t) .
$$

The output measurement available for feedback control is corrupted by noise. In particular, the vector of measured plant outputs $\tilde{y}(t)$ is given by $\tilde{y}(t)=y(t)+v(t)$, where $v(t)=\left[v_{1}(t), \ldots, v_{n_{y}}(t)\right]^{T}$ and $v_{j}(t), j=1, \ldots, n_{y}$ are the individual measurement noise terms that affect each of the plant outputs.

Assumption 1 (Prior assumption on disturbance and noise) $d$ and $v$ are bounded as:

$$
\begin{aligned}
& \left|d_{j}(t)\right| \leq \epsilon_{d_{j}} \\
& \left|v_{j}(t)\right| \leq \epsilon_{v_{j}}
\end{aligned}, \forall t \in \mathbb{Z}, \forall j=1, \ldots, n_{y}
$$

where $\epsilon_{d_{j}}$ and $\epsilon_{v_{j}}$ are positive scalars.

We further assume that the rate of change of the plant parameters, indicated as $\Delta H(t)^{d}$ oteq $H(t)-H(t-1)$, is limited.

Assumption 2 (Bounds on parameter rate of change)

$\Delta H(t) \in\left\{M \in \mathbb{R}^{n_{y} \times m}: K_{j} M_{j} \leq l_{j}, j=1, \ldots, n_{y}\right\}, \forall t \in \mathbb{Z}$,

where $K_{j} \in \mathbb{R}^{n_{\Delta_{j}} \times m}$ and $l_{j} \in \mathbb{R}^{n_{\Delta_{j}}}, j=1, \ldots, n_{y}$ are chosen matrices and vectors defining $n_{\Delta_{j}}$ linear inequalities, forming a bounded set.

Assumption 3 (Bounds on parameter values) The plant model parameters belong to the following set at all times: $H(t) \in \Omega, \forall t \in \mathbb{Z}$, with

$$
\Omega \doteq\left\{H \in \mathbb{R}^{n_{y} \times m}: A_{j 0} H_{j} \leq b_{j 0}, j=1, \ldots, n_{y}\right\},
$$

where the inequalities in (7) should be interpreted as element-wise, and each matrix $A_{j 0} \in \mathbb{R}^{r_{j 0} \times m}$ and vector $b_{j 0} \in \mathbb{R}^{r_{j 0}}$ define a polytope with $r_{j 0}$ faces.

The control objective is to track a given output reference and reject disturbances over a possibly very long time horizon $T(T \gg m)$, while enforcing affine input and output constraints:

$$
\begin{aligned}
& \min _{u(0), \ldots, u(T)} \sum_{t=0}^{T}\left(y(t)-y_{\text {des }}(t)\right)^{T} Q\left(y(t)-y_{\text {des }}(t)\right) \\
&+u(t)^{T} S u(t)+\Delta u(t)^{T} R \Delta u(t) \\
& \text { Subject to, }, \forall t \in[0, T] \\
& C_{u} u(t) \quad \leq g_{u} \\
& C_{\Delta u} \Delta u(t) \leq g_{\Delta u} \\
& C_{y} y(t) \quad \leq g_{y}
\end{aligned}
$$

where $y_{\text {des }}(t) \in \mathbb{R}^{n_{y}}$ is the desired output reference, $Q, S$ and $R$ are positive semi-definite weighting matrices of suitable dimensions selected by the control designer, and $\Delta u(t)=u(t)-u(t-1)$ is the rate of change of the control input. The element-wise inequalities in (8b) define a number $n_{u}, n_{\Delta u}$ and $n_{y}$ of linear constraints on the inputs, input rates, and outputs, respectively. We assume that the set defining the constraints on $\Delta u(t)$ contains the origin, and that the constraint set on $u(t)$ is compact.

Remark 3.1 The considered settings and assumptions hold in a large variety of practical problems. In fact, under the assumption of asymptotic stability, one can embed the effects of truncating an IIR model in the additive disturbance acting on the output of the resulting FIR model. In this way, the considered model class can describe the dynamic behavior of any asymptotically stable linear system. Boundedness of the modeling error due to truncation is guaranteed by the bounds on the input and the decay rate of the impulse response. Regarding Assumptions 2 and 3, these are 
reasonable in practice, when physical insight and prior information on the plant are available. For an example of how to construct the set $\Omega$ for a realistic problem of building climate control, the interested reader is referred to [19]. A typical approach in case of FIR structure entails computing or assuming maximum and minimum values of each FIR coefficient and of its rate of change, in order to build the polytopes in (6)-(7). Note that the set $\Omega$ needs not to be tight, and it can be set to be very large, such that a large number of models is covered. In fact, this set is instrumental to guarantee recursive feasibility and does not affect much the performance. The collected input-output data will provide much tighter parameter bounds, which are adapted in real-time, than the fixed set $\Omega$. Similarly, the bounds on the parameters' change between two time steps and on the signals $d(t)$ and $v(t)$ need not to be known precisely: an overapproximation does not impair our theoretical guarantees. However, assuming too large bounds can lead to excessively cautious control and performance degradation. In principle these bounds can be estimated on-line as well: this is a subject of future research.

\section{Adaptive control algorithm and its properties}

The optimization problem (8) is generally intractable. As a feasible approximate solution, we propose the use of a receding horizon control policy that relies on two steps: 1) a recursive set membership identification that tracks the FPSs, and 2) a model predictive controller that exploits the model set to robustly enforce constraints while optimizing the plant behavior. We now describe in detail these two main steps.

\subsection{Recursive set membership identification algorithm}

Under the working assumptions, each new measurement collected from the plant at time step $t$ delimits a set to which the parameter matrix $H(t)$ is guaranteed to belong:

$\mathcal{S}_{t}(t)=\left\{\begin{array}{c}H \in \mathbb{R}^{n_{y} \times m}:\left|H_{j}^{T} \varphi(t)-\tilde{y}_{j}(t)\right| \leq \epsilon_{d_{j}}+\epsilon_{v_{j}}, \\ j=1, \ldots, n_{y}\end{array}\right\}$

where $\mathcal{S}_{i}(j)$ denotes the set that is defined by the regressor and output measurement vectors at time step $i$, i.e. $\varphi(i)$ and $\tilde{y}(i)$, and that is guaranteed to contain the model parameter matrix $H(j)$ at time step $j$. Geometrically, the set $\mathcal{S}_{t}(t)$ is formed by $2 n_{y}$ linear inequalities defined by the regressor $\varphi(t)$ and the output measurements $\tilde{y}_{j}(t), j=1, \ldots, n_{y}$ collected at time $t$. In addition, we note that the relation between the model parameter matrix at time step $t, H(t)$, and the regressor and plant output vectors at time step $t-1$, i.e. $\varphi(t-1)$ and $y(t-1)$, can be expressed by the following equation:

$$
y(t-1)=H(t) \varphi(t-1)+d(t-1)+\vartheta(t-1),
$$

where $\vartheta(t-1) \in \mathbb{R}^{n_{y}}, \vartheta(t-1)=\left[\vartheta_{1}(t-1), \ldots, \vartheta_{n_{y}}(t-\right.$ $1)]^{T}$, and $\vartheta_{j}(t-1) \in \mathbb{R}, j=1, \ldots, n_{y}$ are the contributions of the unmodeled dynamics to the individual plant outputs.
These terms are present because the parameter matrix $H(t)$ is used in (10) instead of $H(t-1)$ in order to relate the regressor $\varphi(t-1)$ to the output $y(t-1)$ :

$$
\vartheta(t-1) \doteq(H(t-1)-H(t)) \varphi(t-1) .
$$

From Assumption 2, it follows that the signal $\vartheta(t-1)$ is bounded by:

$$
\underline{\vartheta}_{j}(t-1) \leq \vartheta_{j}(t-1) \leq \bar{\vartheta}_{j}(t-1), j=1, \ldots, n_{y},
$$

where the bounds $\underline{\vartheta}_{j}(t-1) \in \mathbb{R}$ and $\bar{\vartheta}_{j}(t-1) \in \mathbb{R}$, $j=1, \ldots, n_{y}$ are the solution to the following two linear programs (LPs):

$$
\begin{aligned}
& \underline{\vartheta}_{j}(t-1) \doteq \min _{x \in \mathbb{R}^{m}} \varphi^{T}(t-1) x \\
& \bar{\vartheta}_{j}(t-1) \doteq \max _{x} \varphi^{T}(t-1) x
\end{aligned}
$$

Subject to:

$$
K_{j} x \leq l_{j}
$$

Based on these definitions, the set $\mathcal{S}_{k}(t)$ is formed on the basis of the regressor and output measured at time step $k \leq t$, i.e. $\varphi(k)$ and $\tilde{y}(k)$, as:

$$
\mathcal{S}_{k}(t)=\left\{\begin{array}{l}
H \in \mathbb{R}^{n_{y} \times m}: \\
\epsilon_{d_{j}}-\epsilon_{v_{j}}+(t-k) \underline{\vartheta}_{j}(k) \leq H_{j}^{T} \varphi(k)-\tilde{y}_{j}(k), \\
H_{j}^{T} \varphi(k)-\tilde{y}_{j}(k) \leq \epsilon_{d_{j}}+\epsilon_{v_{j}}+(t-k) \bar{\vartheta}_{j}(k), \\
j=1, \ldots n_{y}
\end{array}\right\} .
$$

This set is guaranteed to contain the matrix of true system parameters at time step $t, H(t)$. Based on (14) and Assumptions 1-3, we can now define the FPS at time step $t$, denoted by $\mathcal{F}(t)$, as the one containing all parameter matrices $H(t)$ consistent with the prior assumptions and the output measurements collected up to time step $t$ :

$$
\mathcal{F}(t) \doteq \Omega \cap\left(\bigcap_{k=1, \ldots, t} \mathcal{S}_{k}(t)\right)
$$

$\mathcal{F}(t)$ is defined by polytopic constraints on the rows of the model parameter matrix $H(t)$. Thus, it can be uniquely described by a set of matrices and vectors:

$$
\mathcal{F}(t)=\left\{H \in \mathbb{R}^{n_{y} \times m}: A_{j}(t) H_{j} \leq b_{j}(t)\right\},
$$

where each of the matrices and vectors $A_{j}(t) \in \mathbb{R}^{r_{j}(t) \times m}$, $b_{j}(t) \in \mathbb{R}^{r_{j}(t)}, j=1, \ldots, n_{y}$ define $r_{j}(t)$ linear inequalities. Tools to compute automatically $A_{j}(t)$ and $b_{j}(t)$ are available, see e.g. [9]. Note that, as time progresses, the bounds contributed by older data (i.e. the sets $\mathcal{S}_{k}(t)$ with $k \ll t$ ) grow larger and larger, i.e. they naturally become loose.

To use the defined FPS $\mathcal{F}(t)$ for on-line control computation, 
a recursive update approach is needed. To this end, we note that the matrix $A_{j}(t)$ can be derived from $A_{j}(t-1), j=$ $1, \ldots, n_{y}$ by appending two rows formed by the regressor vector at time step $t, \varphi(t)$ and that the vector $b_{j}(t)$ can be formed from $b_{j}(t-1), j=1, \ldots, n_{y}$, by first adding the terms that should account for the possible change of the plant model with respect to the previous time step, and then by appending two new elements that define the constraints related to the newly collected output measurement $\tilde{y}_{j}(t), j=1, \ldots, n_{y}$ :

$$
A_{j}(t)=\left[\begin{array}{c}
A_{j}(t-1) \\
-\varphi^{T}(t) \\
\varphi^{T}(t)
\end{array}\right], b_{j}(t)=\left[\begin{array}{c}
b_{j}(t-1)+\Delta b_{j}(t-1) \\
-\tilde{y}_{j}(t)+\epsilon_{d_{j}}+\epsilon_{v_{j}} \\
\tilde{y}_{j}(t)+\epsilon_{d_{j}}+\epsilon_{v_{j}}
\end{array}\right] .
$$

In (17) the vectors $\Delta b_{j}(t-1) \in \mathbb{R}^{r_{j}(t-1)}, j=1, \ldots, n_{y}$ contain the bounds on the output perturbation induced by all the possible changes of the model dynamics from one time step to the next:

$$
\Delta b_{j}(t-1)=\left[\mathbf{0}_{r_{j o}},-\underline{\vartheta}_{j}(0), \bar{\vartheta}_{j}(0), \ldots,-\underline{\vartheta}_{j}(t-1), \bar{\vartheta}_{j}(t-1)\right]^{T}
$$

with $\mathbf{0}_{r_{j o}} \in \mathbb{R}^{r_{j 0}}$ denoting a vector of $r_{j o}$ zeros.

Using the recursive equation (17) would result, in general, in a growth of the dimensions $r_{j}(t), j=1, \ldots, n_{y}$ by two with each new output measurement. In this way, storing the matrices $A_{j}(t)$ and vectors $b_{j}(t)$ over time would become intractable. Therefore, in order to have a tractable recursive identification algorithm, we keep track of the constraints generated by the most recent $M$ measurements, where $M$ is an even number and a design parameter. In this way the dimensions of the matrices $A_{j}(t)$ and the vectors $b_{j}(t)$ remain bounded over time, such that $r_{j}(t) \leq r_{0 j}+M, \forall j=$ $1, \ldots, n_{y}, \forall t$. The parameter $M$ should be selected such that a good trade-off between conservativeness and computational complexity is reached.

Based on the elements introduced so far, Algorithm 1 summarizes the proposed recursive SM identification algorithm.

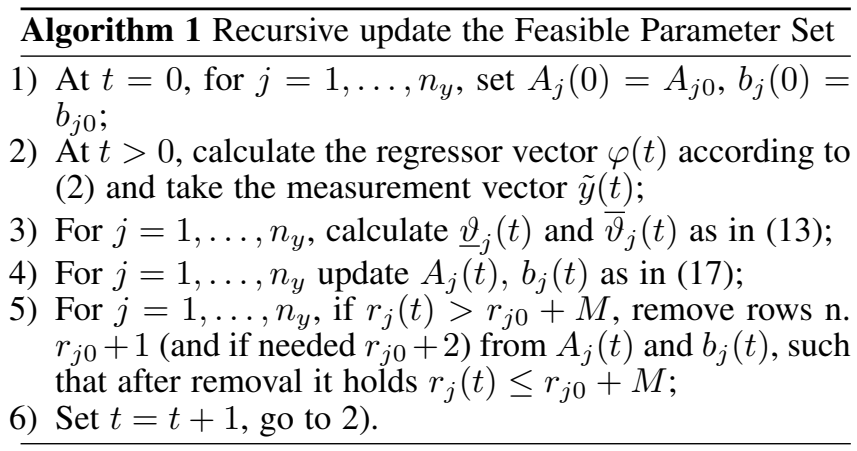

From the derived FPS, we compute a nominal model of the plant at each time step. This is given by a matrix $H_{c}(t) \in \mathbb{R}^{n_{y} \times m}, H_{c}=\left[H_{c, 1}, \ldots H_{c, n_{y}}\right]^{T}$, where the vectors $H_{c, j}(t) \in \mathbb{R}^{m}, j=1, \ldots, n_{y}$ are calculated by solving an LP that aims to find the point inside the FPS closest to the nominal model in the previous time step (i.e. $H_{c}(t-1)$ ):

$$
\min _{H_{c, j}(t), j=1, \ldots, n_{y}} \sum_{j=1}^{n_{y}}\left\|H_{c, j}(t-1)-H_{c, j}(t)\right\|_{1}
$$

Subject to:

$$
A_{j}(t) H_{c j}(t) \leq b_{j}(t), \forall j=1, \ldots, n_{y} .
$$

The matrix $H_{c}(0)$ can be initialized as an arbitrary nonzero element inside the set $\Omega$.

\subsection{Finite horizon optimal control problem}

Let $u(k \mid t), k \in[t, t+N-1], N \geq m$, be candidate future control moves, where the notation $k \mid t$ indicates the prediction at step $k \geq t$ given the information at the current step $t$. For brevity, we collect these decision variables in vector $U \doteq\left[u(t \mid t)^{T} \ldots u(t+N-1 \mid t)^{T}\right]^{T}$. We also define the vectors of future input increments $\Delta u(k \mid t), k \in[t, t+N-1]$ as:

$$
\Delta u(k \mid t)= \begin{cases}u(t \mid t)-u(t-1) & \text { if } k=t \\ u(k \mid t)-u(k-1 \mid t) & \text { if } t+1 \leq k \leq t+N-1\end{cases}
$$

Moreover, we define the future regressor vectors $\varphi(k \mid t) \in$ $\mathbb{R}^{m}, k \in[t+1, t+N]$ as:

$$
\varphi(k \mid t)= \begin{cases}F \varphi(t)+G u(t \mid t) & \text { if } k=t+1 \\ F \varphi(k-1 \mid t)+G u(k-1 \mid t) & \text { if } t+2 \leq k \leq t+N .\end{cases}
$$

Finally, we define the current prediction error $\hat{d}(t) \in \mathbb{R}^{n_{y}}$ as:

$$
\hat{d}(t) \doteq \tilde{y}(t)-H_{c}(t) \varphi(t) .
$$

Then, we consider the following cost function:

$$
\begin{aligned}
& J(U, \tilde{y}(t), \varphi(t)) \doteq \\
& \sum_{k=t}^{t+N-1}\left(\hat{y}(k+1 \mid t)-y_{\mathrm{des}}(k+1 \mid t)\right)^{T} Q(\hat{y}(k+1 \mid t) \\
& \left.-y_{\mathrm{des}}(k+1 \mid t)\right)+u(k \mid t)^{T} S u(k \mid t)+\Delta u(k \mid t)^{T} R \Delta u(k \mid t),
\end{aligned}
$$

where $\hat{y}(k+1 \mid t)=H_{c}(t) \varphi(k+1 \mid t)+\hat{d}(t)$. In (22), $\tilde{y}(t)$ and $\varphi(t)$ are known parameters and $y_{\text {des }}(k \mid t), k \in[t+1, t+N]$, are the predicted values of the desired output. Note that, if the nominal model of the plant $H_{c}(t)$ were equal to the real plant, which would not change in the considered time horizon, the measurement noise $v(t)$ were zero, and the output disturbance $d(t)$ were constant, for $N=T$, minimizing the cost function (22) would be equivalent to minimizing the cost function of the control objective (8).

Satisfaction of input constraints can be enforced by the following set of inequalities:

$$
\begin{aligned}
C_{u} u(k \mid t) & \leq \quad g_{u} \\
C_{\Delta u} \Delta u(k \mid t) & \leq \quad g_{\Delta u}
\end{aligned} \forall k \in[t, t+N-1]
$$


To define the output constraints considered in our MPC design, we first introduce the notion of predicted FPSs, denoted by $\mathcal{F}(k \mid t), k \in[t+1, t+N]$. These sets are computed by propagating the FPS $\mathcal{F}(t)$ in the future, considering the bounds on the rate of change of the parameters. This is done by using the recursive identification Algorithm 1 at each predicted time step, but without considering any future output measurements, which are unknown at the current time:

$$
\mathcal{F}(k \mid t)=\left\{H \in \mathbb{R}^{n_{y} \times m}: A_{j}(k \mid t) H_{j} \leq b_{j}(k \mid t)\right\} .
$$

The predicted matrices $A_{j}(k \mid t)$ and the vectors $b_{j}(k \mid t)$, for $k \in[t+1, t+N-1]$ and $j=1, \ldots, n_{y}$ are initialized as $A_{j}(t \mid t)=A_{j}(t), b_{j}(t \mid t)=b_{j}(t)$ and computed by the recursion:

$$
A(k+1 \mid t)= \begin{cases}A(k \mid t) & \text { if } r_{j}(k \mid t) \leq M^{\prime} \\
{\left[\begin{array}{c}
a_{j 1}(k \mid t) \\
\vdots \\
a_{j r_{j 0}}(k \mid t) \\
a_{j r_{j 0}+3}(k \mid t) \\
\vdots \\
a_{j r_{j}(t)}(k \mid t)
\end{array}\right]} & \text { otherwise, }\end{cases}
$$

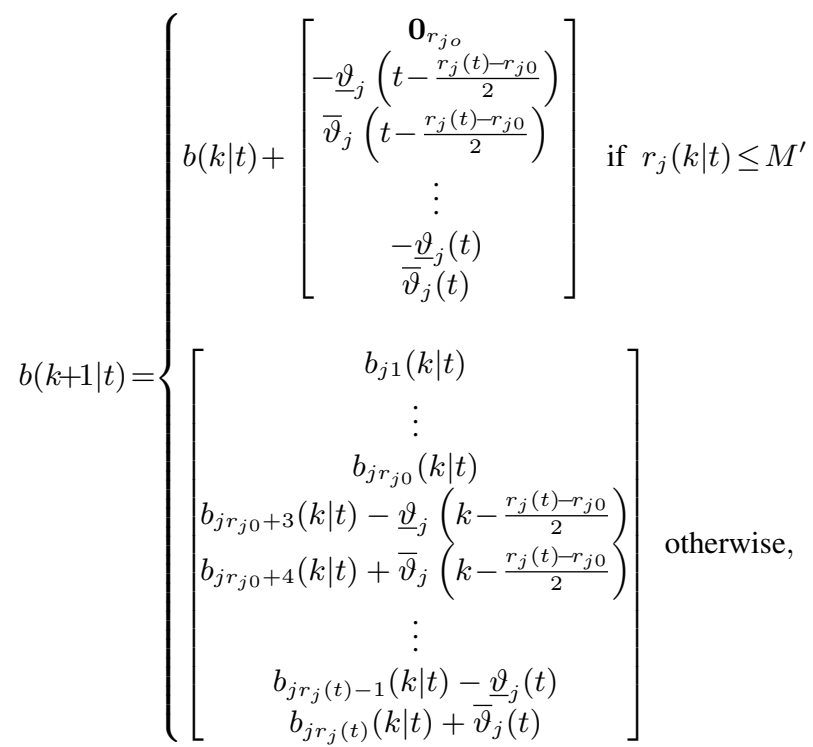

where $a_{j i}(k \mid t)$ and $b_{j i}(k \mid t)$ denote the $i^{\text {th }}$ row of the matrix $A_{j}(k \mid t)$ and the vector $b_{j}(k \mid t)$ respectively, $r_{j}(k \mid t)=$ $r_{j}(t)+2(k-t)$ represents the predicted dimension of the matrices $A_{j}(k)$ and the vectors $b_{j}(k)$ that would be obtained by using Algorithm 1 if no rows would be removed (i.e. if the dimension of the matrices and vectors would be allowed to grow without limit in the future). Finally, the terminal predicted FPS, $\mathcal{F}(t+N \mid t)$, is equal to the set $\Omega$, to which the model parameters are guaranteed to belong at all times.
Remark 4.1 The choice $\mathcal{F}(t+N \mid t)=\Omega$ in principle introduces additional conservativeness, since the set $\mathcal{F}(t+N \mid t)$ could be calculated from the set $\mathcal{F}(t+N-1 \mid t)$ in the same way as for the sets $\mathcal{F}(k \mid t), k \in[t+1, t+N-1]$, and in general such a set would be tighter than the set $\Omega$. However, this approach enables recursive feasibility and robust constraint satisfaction (see Theorem 4.1 later on). Moreover, the impact on the performance is rather small if a long enough prediction horizon is used, as we show in the numerical example of Section 5.

Robust satisfaction of the output constraints is guaranteed by enforcing them for all the parameters inside the predicted FPSs $\mathcal{F}(k \mid t), k \in[t+1, t+N]$ and for all disturbance realizations:

$$
C_{y} H \varphi(k \mid t)+\bar{d} \leq g_{y}, \forall H \in \mathcal{F}(k \mid t), \forall k \in[t+1, t+N],
$$

where $\bar{d}=\left[\bar{d}_{1}, \ldots, \bar{d}_{n_{o}}\right]^{T}$, and $\bar{d}_{l} \in \mathbb{R}, l=1, \ldots, n_{o}$ are given as:

$$
\bar{d}_{l}=\sum_{j=1}^{n_{y}}\left|c_{l j}\right| \epsilon_{d_{j}},
$$

where $c_{l j}$ stands for the element of the $l^{\text {th }}$ row and $j^{\text {th }}$ column of the matrix $C_{y}$. Constraints (27) can be reformulated into a set of linear equality and inequality constraints by using Lemma 3.2 from [18].

To guarantee recursive feasibility, we finally introduce an additional generalized terminal equality constraint, as done e.g. in [7]:

$$
\varphi(t+N \mid t)=F \varphi(t+N \mid t)+G u(t+N-1 \mid t) .
$$

This means that we require the terminal regressor to correspond to a steady state for the considered model structure. Combined with (27), this guarantees robust output constraint satisfaction on an infinite horizon.

For fixed values of $N, Q, S$ and $R$, we can now define the finite horizon optimal control problem (FHOCP) to be solved at each time step $t$ :

$$
\begin{aligned}
& \min _{U, \Lambda} J(U, \tilde{y}(t), \varphi(t)) \\
& \quad \text { Subject to: (23), (27), (28), }
\end{aligned}
$$

which (by using Lemma 3.2 from [18]) can be converted into a quadratic program (QP). The number of decision variables and constrains of this QP depends on the chosen prediction horizon $N$ and the dimension of matrices and vectors that define the FPS $\mathcal{F}(t)$. Its computational complexity can be decreased by reducing the tuning parameter $M$, which bounds the dimension of matrices $A_{j}(t)$ and the vectors $b_{j}(t), j=1, \ldots, n_{y}$, at the cost of higher conservativeness.

\subsection{Properties of the proposed adaptive control algorithm}

The described approach guarantees recursive feasibility and robust satisfaction of both input and output constraints. We first state two results that are instrumental to prove the main one. All the proofs are included in the Appendix. 
Lemma 4.1 Let Assumptions 1-3 hold. Then, the feasible parameter set $\mathcal{F}(t)$ obtained by using the recursive Algorithm 1 is guaranteed to contain the true model parameter matrix at each time step, i.e. $\mathcal{F}(t) \neq \emptyset$ and $H(t) \in$ $\mathcal{F}(t), \forall t \geq 0$.

Lemma 4.2 Let Assumptions 1-3 hold. Then, when Algorithm 1 is used, at each time step $t$, it holds that $\mathcal{F}(k \mid t+1) \subseteq$ $\mathcal{F}(k \mid t), k \in[t+2, t+N]$.

We now state the main result related to recursive feasibility of the finite horizon optimal control problem and robust constraint satisfaction.

Theorem 4.1 Let Assumptions 1-3 hold, and assume that the problem (29) is feasible at time $t=0$. Then the problem (29) is recursively feasible and the closed-loop system obtained by applying the proposed adaptive algorithm is guaranteed to satisfy input and output constraints $\forall t \geq 0$.

Remark 4.2 With respect to our previous contribution [18], the key technical additions that allow us to guarantee recursive feasibility (hence robust constraint satisfaction) also in the LTV case are: 1) the inflation of the FPS both with respect to past data (see (14)) and future time (see (25)-(26); and 2) the use of robust output constraints at the end of the prediction horizon with respect to the whole set $\Omega$. Both these measures generally increase the conservativeness of the adaptive MPC algorithm, this is the price to pay to obtain guaranteed properties. As mentioned (see Remark 4.1), such conservativeness can be mitigated by increasing the prediction horizon $N$. In this way, the presence of the terminal constraint does not have a large impact on the performance at the beginning of the prediction horizon, which is the part that impacts the close-loop behavior. Finally, one can show that if the nominal model parameters do not change over a given time period, then during such an interval the approach exhibits integral action. The result is omitted since it is a minor modification of Lemma 4.1 in [18].

Remark 4.3 It is interesting to compare our approach with $M P C$ techniques for Linear Parameter-Varying $(L P V)$ systems. In LPV-MPC, the system is known a priori, meaning that for a given value of the scheduling parameter the dynamics are known. The scheduling parameter might be measured or not. In the first (most frequent) case (see e.g. [5]), the past and current system dynamics are known, and the predictions need to account for changes in the scheduling parameter, which results in a change of the system dynamics. In the second case, the past and current dynamics are not exactly known and one would need to estimate the scheduling parameter. Differently from either setup, in our work we assume that the time-varying dynamics are not known. Our setup is equivalent to an LPV one with unmeasured scheduling parameters, where all the coefficients of the system matrices are time-varying parameters that need to be estimated from data. Moreover, we employ a robust approach, meaning that we estimate on-line not only a nominal value of the parameters, but also all possible system parameters consistent with the measured data, and we robustify the design against such uncertainty.

\section{Simulation results - motivating example revisited}

To illustrate its effectiveness, we apply the new approach to the example of Section 2. We take the set $\Omega$ as in Fig. 1. We use a FIR model structure with $m=24$ and assume the bounds on $\Delta H(t)$ shown in Fig. 4. The actual values of $\Delta H(t)$ during the simulation are presented in Fig. 4 as well, showing that it is not required that the assumed bounds are tight or known exactly. We set the MPC prediction horizon to 30. Fig. 5 shows the obtained results when the proposed algorithm is used. As it can be seen, the new approach copes with the change of the actual plant parameters, satisfying robustly the constraints, and the actual plant output is always inside the estimated uncertainty bounds, meaning that the true plant parameters are inside the FPS despite their time varying nature. Very good tracking performance are achieved, too. Regarding this aspect, we also evaluated the effect of the robust terminal constraint on the conservativeness of the control algorithm (see Remark 4.2), by repeating the simulation without such a constraint. In cases when the optimal control problem was not feasible, we applied the last calculated control input. During the simulation there were actually 4 time steps in which infeasibility occurred. On the other hand, the obtained control performance was extremely similar, with just a $3 \%$ reduction of the mean square tracking error with respect to the one obtained in presence of the terminal constraint. Another simulation study, considering a multivariable three-tank system, is available in [17], showing the advantages with respect to an adaptive MPC approach that relies on a certainty equivalence model identification strategy.

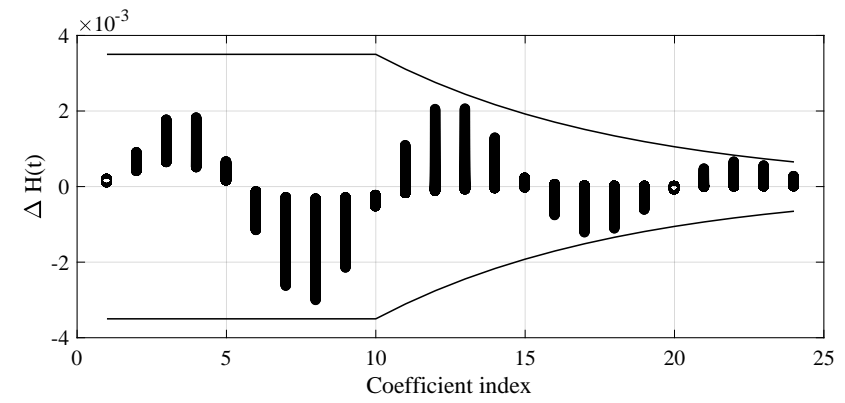

Fig. 4. Bounds on the rate of change of the impulse response coefficients (solid line) and actual values of $\Delta H(t)$ during the simulation (circles).

\section{Conclusion}

We described an adaptive MPC algorithm for asymptotically stable, constrained LTV systems with multiple inputs and outputs. The technique relies on a novel recursive SM identification approach to keep track of the set of all possible model parameters that are consistent with data and with the prior assumptions. The recursive update of such a set accounts for the time-varying nature of the system through assumed (not necessarily tight) bounds on the parameters' rate of change. The MPC design guarantees recursive feasibility and robust satisfaction of output constraints. A simulation study shows that the new algorithm removes a fundamental limitation of a previously proposed adaptive MPC approach. 


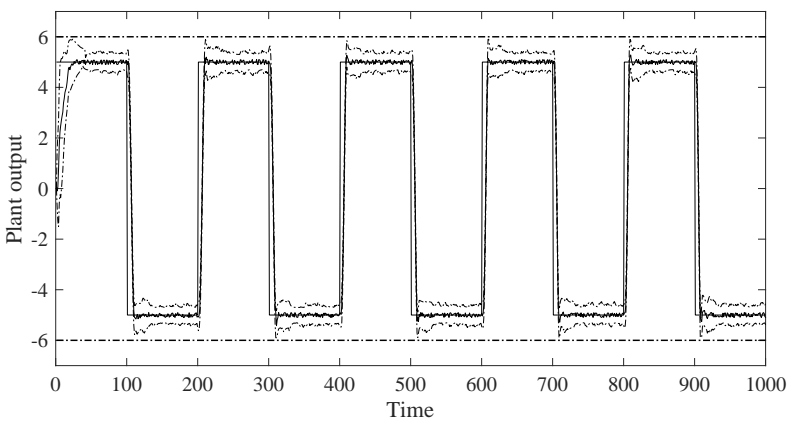

Fig. 5. Plant output (thick black line), reference (dashed line), uncertainty bounds (dotted lines) and constraints (dash-dotted lines) with the proposed adaptive MPC algorithm.

\section{Appendix}

Proof of Lemma 4.1. We use induction to prove the claim of the Lemma. At time step $t=0$, from the step 1) of Algorithm 1, it holds that $\mathcal{F}(0)=\Omega$ and from Assumption 3, it then follows that $\mathcal{F}(0) \neq \emptyset$ and that $H(0) \in \mathcal{F}(0)$. Let us now, for the sake of the inductive argument, assume that at some time step $t \geq 0$, it holds that $H(t) \in \mathcal{F}(t)$. We shall show, that it than follows that $H(t+1) \in \mathcal{F}(t+1)$. To this end, we define matrices $A_{j}^{\prime}(t) \in \mathbb{R}^{r_{j 0} \times m}, A_{j}^{\prime \prime}(t) \in \mathbb{R}^{\left(r_{j}(t)-r_{j 0}\right) \times m}$ and vectors $b_{j}^{\prime}(t) \in$ $\mathbb{R}^{r_{j 0}}, b_{j}^{\prime \prime}(t) \in \mathbb{R}^{r_{j}(t)-r_{j 0}}, j=1, \ldots, n_{y}$, as:

$$
\begin{gathered}
b^{\prime}(t)=\left[\begin{array}{c}
b_{j 1} \\
\vdots \\
b_{j r_{j 0}}
\end{array}\right], b^{\prime \prime}(t)=\left[\begin{array}{c}
b_{j r_{j 0}+1} \\
\vdots \\
b_{j r_{j}(t)}
\end{array}\right], A_{j}^{\prime}(t)=\left[\begin{array}{c}
a_{j 1} \\
\vdots \\
a_{j r_{j 0}}
\end{array}\right], \\
A_{j}^{\prime \prime}(t)=\left[\begin{array}{c}
a_{j r_{j 0}+1} \\
\vdots \\
a_{j r_{j}(t)}
\end{array}\right]=\left[\begin{array}{c}
-\varphi^{T}\left(t-\frac{r_{j}(t)-r_{j 0}}{2}\right) \\
\varphi^{T}\left(t-\frac{r_{j}(t)-r_{j 0}}{2}\right) \\
\vdots \\
-\varphi^{T}(t) \\
\varphi^{T}(t)
\end{array}\right]
\end{gathered}
$$

Note that $A_{j}(t)=\left[\begin{array}{c}A_{j}^{\prime}(t) \\ A_{j}^{\prime \prime}(t)\end{array}\right]$ and $b_{j}(t)=\left[\begin{array}{c}b_{j}^{\prime}(t) \\ b_{j}^{\prime \prime}(t)\end{array}\right]$. From Assumption 3 , it holds that:

$$
A_{j}^{\prime}(t) H_{j}(t+1) \leq b_{j}^{\prime}(t), j=1, \ldots, n_{y} .
$$

In addition, we note that from the inductive assumptions, it holds that $A_{j}^{\prime \prime}(t) H_{j}(t) \leq b_{j}^{\prime \prime}(t), j=1, \ldots, n_{y}$. Therefore, it than also holds that:

$$
A_{j}^{\prime \prime}(t) H_{j}(t+1) \leq b_{j}^{\prime \prime}(t)+e_{j}(t)
$$

where $e_{j}(t) \in \mathbb{R}^{r_{j}(t)-r_{j 0}}, e_{j}(t)=A_{j}^{\prime \prime}(t)\left(H_{j}(t+1)-H_{j}(t)\right)$, $j=1, \ldots, n_{y}$. From the definition of $A_{j}^{\prime \prime}(t)$ (note that this matrix is exclusively formed from the past regressor vectors), and the definition of $\bar{\vartheta}(t)$ and $\underline{\vartheta}(t)$ in (13), we note that the vectors $e_{j}(t), j=1, \ldots, n_{y}$ are bounded such that it holds $e_{j}(t) \leq \bar{e}_{j}$, where:

$\bar{e}_{j}=\left[-\underline{\vartheta}\left(t-\frac{r_{j}(t)-r_{j 0}}{2}\right), \bar{\vartheta}\left(t-\frac{r_{j}(t)-r_{j 0}}{2}\right), \ldots,-\underline{\vartheta}(t), \bar{\vartheta}(t)\right]^{T}$
Therefore, it holds that:

$$
A_{j}^{\prime \prime}(t) H_{j}(t+1) \leq b_{j}^{\prime \prime}(t+1)+\bar{e}_{j}, j=1, \ldots, n_{y} .
$$

Moreover, from Assumption 1, it holds that the following two inequalities have to be satisfied:

$$
\begin{aligned}
& -\varphi(t+1) H_{j}(t+1) \leq-\tilde{y}_{j}(t+1)+\epsilon_{d_{j}}+\epsilon_{v_{j}} \\
& -\varphi(t+1) H_{j}(t+1) \leq \tilde{y}_{j}(t+1)+\epsilon_{d_{j}}+\epsilon_{v_{j}}
\end{aligned}
$$

Based on (30), (31) and (32), it holds that:

$$
A_{j}^{\dagger}(t+1) H_{j}(t+1) \leq b_{j}^{\dagger}(t+1), j=1, \ldots, n_{y},
$$

where

$$
A_{j}^{\dagger}(t+1)=\left[\begin{array}{c}
A_{j}^{\prime}(t) \\
A_{j}^{\prime \prime}(t) \\
-\varphi(t+1) \\
\varphi(t+1)
\end{array}\right], b_{j}^{\dagger}(t+1)=\left[\begin{array}{c}
b_{j}^{\prime}(t) \\
b_{j}^{\prime \prime}(t+1)+\bar{e}_{j} \\
-\tilde{y}_{j}(t+1)+\epsilon_{d_{j}}+\epsilon_{v_{j}} \\
\tilde{y}_{j}(t+1)+\epsilon_{d_{j}}+\epsilon_{v_{j}}
\end{array}\right]
$$

are the matrices that would be obtained after running the step 4) of Algorithm 1 at time $t+1$ (i.e. before removing any rows from the matrices and vectors in order to keep their dimensions bounded). Therefore, the set $\mathcal{F}^{\dagger}(t+1)=$ $\left\{H \in \mathbb{R}^{n_{y} \times m}: A_{j}^{\dagger}(t+1) H_{j} \leq b_{j}^{\dagger}(t+1)\right\}$ is a nonempty set that is guaranteed to contain $H(t+1)$, i.e. $H(t+1) \in \mathcal{F}^{\dagger}(t+1)$. Set $\mathcal{F}^{\dagger}(t+1)$ represents the updated feasible parameter set before possible removal of any inequalities in order to bound the complexity of its description. The set $\mathcal{F}(t+1)$ is obtained by either taking the set $\mathcal{F}^{\dagger}(t+1)$ as it is (i.e. when $\left.r_{j}(t) \leq M+r_{j 0}, \forall j=1, \ldots, n_{y}\right)$, or by removing several inequalities that constitute it (see step 5) of Algorithm 1). Therefore it holds that $\mathcal{F}^{\dagger}(t+1) \subseteq \mathcal{F}(t+1)$, and hence it holds that $H(t+1) \in \mathcal{F}(t+1)$, which means that $\mathcal{F}(t+1) \neq \emptyset$. By invoking the argument of mathematical induction, it then holds that $H(t) \in \mathcal{F}(t), \forall t \geq 0$, which completes the proof.

Proof of Lemma 4.2. We first note that, from the definition of $\mathcal{F}(t+1 \mid t)$ (see (24),(25) and (26)), and the way Algorithm 1 works, it holds that:

$$
A_{j}(t+1)=\left[\begin{array}{c}
A_{j}(t+1 \mid t) \\
-\varphi(t+1) \\
\varphi(t+1)
\end{array}\right], b_{j}(t+1)=\left[\begin{array}{c}
b_{j}(t+1 \mid t) \\
-\tilde{y}_{j}(t+1)+\epsilon_{d_{j}}+\epsilon_{v_{j}} \\
\tilde{y}_{j}(t+1)+\epsilon_{d_{j}}+\epsilon_{v_{j}}
\end{array}\right] .
$$

Matrices $A_{j}(k \mid t+1)$ and vectors $b_{j}(k \mid t+1), j=1, \ldots, n_{y}$ are then, by construction, formed from the matrices $A_{j}(t+1)$ and $b_{j}(t+1)$. Therefore we have that, for $j=1, \ldots, n_{y}$ and $k \in[t+2, t+N]$, it holds:

$A_{j}(k \mid t+1)=\left[\begin{array}{c}A_{j}(k \mid t) \\ -\varphi(t+1) \\ \varphi(t+1)\end{array}\right], b_{j}(k \mid t+1)=\left[\begin{array}{c}b_{j}(k \mid t) \\ -\tilde{y}_{j}(t+1)+\epsilon_{d_{j}}+\epsilon_{v_{j}} \\ \tilde{y}_{j}(t+1)+\epsilon_{d_{j}}+\epsilon_{v_{j}}\end{array}\right]$

As it holds that $\mathcal{F}(k \mid t)=\left\{H \in \mathbb{R}^{n_{y} \times m}: A_{j}(k \mid t) H_{j} \leq b_{j}(k \mid t)\right\}$, and $\mathcal{F}(k \mid t+1)=\left\{H \in \mathbb{R}^{n_{y} \times m}: A_{j}(k \mid t+1) H_{j} \leq b_{j}(k \mid t+1)\right\}$, 
$\forall k \in[t+2, t+N-1]$, it holds that each of the sets $\mathcal{F}(k \mid t+1), k \in[t+1, t+N-1]$ is formed by the same inequalities as the set $\mathcal{F}(k \mid t)$ and that it has two additional inequalities defined by the regressor vector and output measurement at time step $t+1$. Therefore, it holds that $\mathcal{F}(k \mid t+1) \subseteq \mathcal{F}(k \mid t), k \in[t+2, t+N-1]$. In addition, we note that $\mathcal{F}(t+N \mid t)=\Omega$ and that for $j=1, \ldots, n_{y}$, it holds that:

$$
A_{j}(t+N \mid t+1)=\left[\begin{array}{c}
A_{j 0} \\
A_{j}^{\prime}
\end{array}\right], b_{j}(t+N \mid t+1)=\left[\begin{array}{c}
b_{j 0} \\
b_{j}^{\prime}
\end{array}\right],
$$

where the matrices $A_{j}^{\prime}$ and the vectors $b_{j}^{\prime}, j=1, \ldots, n_{y}$ are obtained by using the rules for generating the predicted matrices $A_{j}(k \mid t)$ and vectors $b_{j}(k \mid t)$ in (25) and (26). Therefore, from the definition of $\mathcal{F}(t+N \mid t+1)$ (see e.g. (24)) and the definition of the set $\Omega$ in (7), it holds that $\mathcal{F}(t+N \mid t+1) \subseteq \mathcal{F}(t+N \mid t)$. Hence, it holds that $\mathcal{F}(k \mid t+1) \subseteq \mathcal{F}(k \mid t), k \in[t+2, t+N]$, which completes the proof.

Proof of Theorem 4.1. We first show that the FHOCP (29) is recursively feasible. To this end, we use induction. The problem (29) is feasible for $t=0$ by assumption. Let us assume that the problem (29) is feasible at a generic time step $t$ and let the optimal control sequence be $U^{*}(t)=\left[u^{*}(t \mid t), \ldots, u^{*}(t+N-1 \mid t)\right]$, and its corresponding sequence of predicted regressor vectors be $\varphi^{*}(k \mid t), k=t+1, \ldots, t+N$. Then, a possible feasible control sequence at $t+1$ is $U(t+1)=\left[u^{*}(t+1 \mid t), \ldots, u^{*}(t+N-\right.$ $\left.1 \mid t), u^{*}(t+N-1 \mid t)\right]$. This sequence satisfies constraints $(23)$ and (28). In addition, we note that the predicted regressor vectors $\varphi(k \mid t+1), k=t+2, \ldots, t+N+1$ that correspond to the input sequence $U(t+1)$, by construction satisfy the equalities $\varphi(k \mid t+1)=\varphi^{*}(k \mid t)$, for $k \in[t+2, t+N]$ and that from (28) it follows that $\varphi(t+N+1 \mid t+1)=\varphi^{*}(t+N \mid t)$. Moreover, we note that from Lemma 4.2, it holds that $\mathcal{F}(k \mid t+1) \subseteq \mathcal{F}(k \mid t), \forall k \in$ $[t+1, t+N]$. In addition, we note that $\mathcal{F}(t+\bar{N}+1 \mid t+1)=$ $\mathcal{F}(t+N \mid t)=\Omega$. Based on this, the sequence of inputs $U(t+1)$ satisfies the output constraints (27), and hence the FHOCP (29) has a feasible solution. Repeating this argumentation for all $t>0$, it can be concluded that the FHOCP (29) remains feasible $\forall t>0$. From this and Lemma 4.1, the other claim of the Theorem follows directly.

\section{References}

[1] V. Adetola, D. DeHan, and M. Guay. Robust adaptive MPC for constrained uncertain nonlinear systems. International Journal of Adaptive Control and Signal Processing, 25:155-168, 2011.

[2] A. Aswani, H. Gonzalez, S. Sastry, and C. Tomlin. Provably safe and robust learning-based model predictive control. Automatica, 49:1216-1226, 2013.

[3] S. Di Cairano. Indirect adaptive model predictive control for linear systems with polytopic uncertainty. In American Control Conference (ACC), 2016, pages 3570-3575, Boston, MA, USA, 2016.

[4] M. Canale, L. Fagiano, and M.C. Signorile. Nonlinear model predictive control from data: a set membership approach. International Journal of Robust and Nonlinear Control, 24(1):123139, 2014.

[5] A. Casavola, D. Famularo, and G. Franze. A feedback min-max mpc algorithm for lpv systems subject to bounded rates of change of parameters. IEEE Transactions on Automatic Control, 47(7):1147$1153,2002$.

[6] P. M. J. Van den Hof, P. S. C. Heuberger, and J. Bokor. System identification with generalized orthonormal basis functions. Automatica, 31:1821-1834, 1995.
[7] L. Fagiano and A. Teel. Generalized terminal state constraint for model predictive control. Automatica, 49:2622-2631, 2012.

[8] T.A. N. Heirung, E. Ydstie, and B. Foss. Dual adaptive model predictive control. Automatica, 80:340-348, 2017.

[9] M. Herceg, M. Kvasnica, C.N. Jones, and M. Morari. MultiParametric Toolbox 3.0. In Proc. of the European Control Conference, pages 502-510, Zürich, Switzerland, July 17-19 2013.

[10] L. Hewing, A. Liniger, and M. N. Zeilinger. Cautious nmpc with gaussian process dynamics for autonomous miniature race cars. In European Control Conference, pages 4029-4036, Limassol, Cyprus, 2018.

[11] T.-K. Kim and T. Sugie. Adaptive receding horizon predictive control for constrained discrete-time linear systems with parameter uncertainties. International Journal of Control, 81:62-73, 2008.

[12] D. Limon, J. Calliess, and J.M. Maciejowski. Learning-based nonlinear model predictive control. In IFAC World Congress 2017, pages 8035 - 8042, Toulouse, France, 2017.

[13] M. Lorenzen, F. Allgöwer, and M. Cannon. Adaptive model predictive control with robust constraint satisfaction. In IFAC World Congress 2017, pages 3313 - 3318, Toulouse, France, 2017.

[14] G. Marafioti, R. Bitmead, and M. Hovd. Persistently exciting model predictive control. Adaptive Control and Signal Processing, 28:536552, 2014.

[15] C. J. Ostafew, A. P. Schoellig, and T. D. Barfoot. Learning-based nonlinear model predictive control to improve vision-based mobile robot path-tracking in challenging outdoor environments. In Robotics and Automation (ICRA), 2014 IEEE International Conference on, pages 4029-4036, Hong Kong, 2014.

[16] S.J. Qin and T.A. Badgwell. A survey of industrial model predictive control technology. Control Engineering Practice, 11:733-764, 2003.

[17] M. Tanaskovic, L. Fagiano, and V. Gligorovski. Adaptive model predictive control for constrained time varying systems. In European Control Conference, pages 1698-1703, Limassol, Cyprus, 2018.

[18] M. Tanaskovic, L. Fagiano, R. Smith, and M. Morari. Adaptive receding horizon control for constrained MIMO systems. Automatica, 50:3019-3029, 2014.

[19] Marko Tanaskovic, David Sturzenegger, Roy Smith, and Manfred Morari. Robust adaptive model predictive building climate control. IFAC-PapersOnLine, 50(1):1871 - 1876, 2017. 20th IFAC World Congress.

[20] B. Wahlberg. System identification using Laguerre models. IEEE Transactions on Automatic Control, 36:551-562, 1991.

[21] B. Wahlberg. System identification using Kautz models. IEEE Transactions on Automatic Control, 39:1276-1282, 1994.

[22] X. Wang, Y. Sun, and K. Deng. Adaptive model predictive control of uncertain constrained systems. In Proceedings of the 2014 American Control Conference, pages 2857 - 2862, Portland, OR, 2014.

[23] J. Zhou, S. Di Cairano, and C. Danielson. Indirect adaptive MPC for output tracking of uncertain linear polytopic systems. In Proceedings of the 2017 American Control Conference, pages 3054 - 3059, Seattle, WA, 2017. 